\title{
Highly selective peripheral nerve radio frequency ablation for the treatment of severe knee osteoarthritis
}

\author{
LIN XIAO $^{1 *}$, FANGWU SHU ${ }^{2 *}$, CHENG XU $^{1}$, ZHONGGUI ZHANG ${ }^{1}$, \\ LIANG HUANG $^{1}$ XIANMO WANG ${ }^{3}$ and YAN ZHANG ${ }^{4}$ \\ ${ }^{1}$ Department of Pain Management, The First People's Hospital of Jingzhou, Jingzhou, Hubei 434000; \\ ${ }^{2}$ Department of Pain Management, Jiangling County People's Hospital, Jiangling, Hubei 434100; \\ ${ }^{3}$ Clinical Laboratory and ${ }^{4}$ Ear, Nose, Throat and Head Neck Surgery Department, \\ The First People's Hospital of Jingzhou, Jingzhou, Hubei 434000, P.R. China
}

Received January 20, 2017; Accepted September 15, 2017

DOI: $10.3892 /$ etm.2018.6658

\begin{abstract}
The objective of the present study was to investigate the effect of highly selective peripheral nerve radiofrequency $(\mathrm{RF})$ ablation for the pain caused by severe knee osteoarthritis (OA). A total of 96 patients with knee OA were randomly divided into two groups. The 49 patients in group A were treated with highly selective peripheral nerve RF ablation group and the 47 patients in group B were treated with sodium hyaluronate injection. Visual analogue scale (VAS), Lysholm knee score (LKS), and pain relief before treatment and at 3 days and 3, 6, 9, and 12 months after treatment were compared. At each time point after treatment in group A, both VAS and LKS scores were significantly different compared with before treatment $(\mathrm{P}<0.05)$. At 3 days, and at 3 and 6 months after treatment in group B, VAS scores were significantly different from before treatment $(\mathrm{P}<0.05)$ and at 9 and 12 months after treatment, the scores were not significantly different from those before treatment. In addition, LKS scores were only significantly different at 3 months after treatment compared with before treatment $(\mathrm{P}<0.05)$ and not significantly different after that time point. Furthermore, compared with group B, the rates of effective pain relief in group A were significantly higher at each time point after treatment $(\mathrm{P}<0.05)$. Compared with sodium hyaluronate injection, highly selective
\end{abstract}

Correspondence to: Dr Xianmo Wang, Clinical Laboratory, The First People's Hospital of Jingzhou, 8 Hangkong Road, Jingzhou, Hubei 434000, P.R. China

E-mail: ehxkmvj424158@163.com

Dr Yan Zhang, Ear, Nose, Throat and Head Neck Surgery Department, The First People's Hospital of Jingzhou, 8 Hangkong Road, Jingzhou, Hubei 434000, P.R. China

E-mail:66552555@qq.com

*Contributed equally

Key words: knee joint, radiofrequency ablation, nerve locator, sodium hyaluronate peripheral nerve RF ablation of the knee was more effective, easy to operate and had no significant adverse effects for the treatment of knee OA.

\section{Introduction}

Knee osteoarthritis (OA) is a disease characterized by chronic articular cartilage lesions $(1,2)$. The main pathological features include degenerative changes to articular cartilage and osteophyte formation at joint edges (3). The clinical manifestations include knee pain, stiffness, movement disorder, knee swelling and deformation (4). At present, there are no methods that effectively block the progression of knee OA and the main goals of treatment are limited to alleviation of pain and functional improvement $(5,6)$. Concerning the current goals of treatment, our department is investigating a new therapeutic method-highly selective denervation to alleviate pain in patients with knee OA, with the aim of exploring an accurate, safe and effective method to treat chronic knee pain.

\section{Patients and methods}

Patients. A total of 96 patients, including 27 males and 69 females treated for knee OA at Jingzhou First People's Hospital (Jingzhou, China) from June 2012 to December 2012 were selected. Patients were aged from $47-70$ years, with average age of 58.2 \pm 5.5 years. A total of 37 patients had OA in the left knee, 27 in the right and 32 in both knees. The course of disease was from 22-54 months, with average of 31.4 \pm 7.9 months. Body weight ranged from $53-97 \mathrm{~kg}$. This study was approved by the hospital Ethics Committee. All patients signed the informed consent and were randomly divided into two groups. There were 49 patients in group A [received highly selective peripheral nerve radiofrequency (RF) ablation] including 12 males and 37 females, with average age of $56.5 \pm 9.5$ years. The average course of disease was $36.5 \pm 7.5$ months. There were 47 patients in group B (received sodium hyaluronate injection) including 11 males and 36 females, with average age of $61.5 \pm 8.5$ years. The average course of disease was $35.5 \pm 6.5$ months. There were no significant differences between the two groups in terms of general parameters, 
Table I. Basic data for the two groups (mean \pm standard deviation).

\begin{tabular}{|c|c|c|c|c|c|c|c|c|c|c|}
\hline \multirow[b]{2}{*}{ Groups } & \multirow[b]{2}{*}{ Cases } & \multicolumn{2}{|c|}{$\operatorname{Sex}(n)$} & \multirow{2}{*}{$\begin{array}{c}\text { Age } \\
\text { (years) }\end{array}$} & \multirow{2}{*}{$\begin{array}{c}\text { Disease } \\
\text { courses } \\
\text { (months) }\end{array}$} & \multicolumn{3}{|c|}{ Diseased region $(\mathrm{n})$} & \multirow{2}{*}{$\begin{array}{l}\text { VAS before } \\
\text { cure (scores) }\end{array}$} & \multirow{2}{*}{$\begin{array}{c}\text { LKS } \\
\text { (scores) }\end{array}$} \\
\hline & & Male & Female & & & Left & Right & Couple & & \\
\hline A & 49 & 12 & 37 & $56.5 \pm 9.5$ & $36.5 \pm 7.5$ & 19 & 13 & 17 & $7.3 \pm 1.2$ & $42.4 \pm 8.2$ \\
\hline B & 47 & 11 & 36 & $61.5 \pm 8.5$ & $35.5 \pm 6.5$ & 18 & 14 & 15 & $7.3 \pm 1.4$ & $41.2 \pm 7.3$ \\
\hline
\end{tabular}

visual analogue scale (VAS) and Lysholm knee score (LKS) $(\mathrm{P}>0.05)$ (Table I).

$\mathrm{OA}$ of the knee was diagnosed with reference to the 1995 revised diagnostic criteria for knee OA of the American College of Rheumatology (San Diego, CA, USA) (1,7) as follows: i) Sounds produced from friction of joint activity; ii) knee pain for $\geq 14$ days within 1 month before visit; iii) knee stiffness in the morning lasting $\leq 30 \mathrm{~min}$; iv) age over 4 years; v) extensive pressure pain points on knees with swelling or twist sense and severe pain; and vi) standing or weight-bearing $\mathrm{X}$-ray showing significantly narrowed knee joint spaces and osteophyte formation at the edges of epiphyses. Patients with criteria $1,2,3,4$, and 5 or 1 and 6 were diagnosed with knee OA.

The inclusion criteria included (7): i) Patients meeting the diagnostic criteria; ii) those with VAS score $>6$; iii) those with joint LKS score $>60$; iv) those who voluntarily abandoned other therapeutic measures during treatment for OA; v) those from 40-70 years old; vi) those who did not take other medicines and undergo other therapeutic methods within the previous month before the study; and vii) those with good compliance and who agreed to sign the informed consent.

The exclusion criteria included: i) Those with significant knee deformity and varus or valgus knees; ii) cases complicated with significant organic pathologic changes, serious cardiovascular diseases, or malignancy; iii) cases with history of neurovascular injuries of the affected extremities; iv) cases complicated with rheumatoid arthritis, osteomyelitis, knee tumors, gout, diapyesis and acute phase intra-articular fractures or neurovascular injuries; v) cases with bone bridge connections formed between joints causing significantly narrowed joint spaces appearing as bony ankylosis; vi) cases with history of knee surgery, skin infection of a specific location, or severe trauma around the knee; vii) those allergic to sodium hyaluronate; viii) those with disease course over 10 years and viiii) those with mental disorders.

Treatment. Patients in group A were treated with highly selective peripheral nerve RF ablation of the knee as follows: Patients were placed in the dorsal position with knees slightly bent with a thin pillow placed on the backside of the affected knees; inspections were performed around the skin of the knee; a peripheral nerve plexus stimulator (multistim sensor; Pajunk ${ }^{\circledR}$ Holding GmbH, Geisingen, Germany) was used to locate peripheral nerves of the knee, focusing on the cutaneous branch of femoral nerves on both sides of the patella and patellar branch of saphenous nerves that were susceptible to pain provocation. After completion of routine sterilization, local anesthesia was performed using $1 \%$ dolicaine. After anesthesia, a $5 \mathrm{~cm}$ RF trocar was used to vertically pierce roughly $1.5 \mathrm{~cm}$ into the cutaneous branch of femoral nerves on both sides of the patella. For the patellar branches of the saphenous nerves, it was required to reach deep into the periosteum, so for thin patients, it was necessary to lift the skin to avoid damage. After the puncture needle was inserted, an RF controlled fuser (model: R-2000B; Beiqi, Beijing, China) was connected. Tests were performed at voltage of $100 \mathrm{~Hz}$ and $0.5 \mathrm{~V}$, respectively. If pain was caused on the corresponding regions of the knee, thermocoagulation ablation was performed, with settings of 60,70 and $80^{\circ} \mathrm{C}$ as the temperature cycle and $90 \mathrm{sec}$ as the RF ablation time cycle. The above steps were repeated to ablate each puncture point. After surgery, conventional treatment was performed.

Patients in group B, were treated with intra-articular injection of sodium hyaluronate (specification, $25 \mathrm{mg} /$ bottle; Chemical Industries Ltd., Japan). A puncture was made on the upper edge of the patella. Local routine disinfection of the skin was performed. During puncturing, patients were placed in the straight dorsal position and the musculus quadriceps were relaxed. The operator wearing gloves moved the patella outward at 1/3 of the upper edge of the patella with the left thumb, while the index finger and middle finger were touching the lower edge of the patella to form an obvious mark. The puncture needle was inserted from spaces and sodium hyaluronate was injected with a sterile syringe into the articular cavity. If it felt empty, and no blood was withdrawn, $25 \mathrm{mg} / 2.5 \mathrm{ml}$ of physic liquor was slowly injected into the articular cavity. After completing the injection and withdrawing the needle, the operator applied pressure to the puncture site using a sterilized cotton ball, and applied paste wound dressing. Patients were instructed not to wet the puncture sites for $24 \mathrm{~h}$ and not to take a bath, to keep the puncture site dry to prevent infection. Patients performed knee exercises to make the medicine distribute evenly within the articular cavity to achieve the effect of pain alleviation. If there were hydrops in the articular cavity before injection of sodium hyaluronate, they were extracted. The injections were alternatively performed on the inner and outer sides of the knee. Injections were made in the articular cavity once per week for five consecutive weeks, consisting one course of treatment. Doctors advised patients to perform non-weight-bearing joint activities and static muscle contraction training.

VAS was used according to the amount of pain felt by the patient. Patients were asked to mark the pain positions between the rider scale bars and make corresponding scores. A score of 0 indicated no pain, and 10 indicated the most severe pain. 
Table II. Knee VAS, LKS scores before and after treatment (mean \pm standard deviation).

\begin{tabular}{lcccccccc}
\hline & & & & \multicolumn{5}{c}{ After treatment } \\
\cline { 7 - 9 } Scores & Groups & Cases & $\begin{array}{c}\text { Before } \\
\text { treatment }\end{array}$ & At 3 day & At 3 months & At 6 months & At 9 months & At 12 months \\
\hline VAS & A & 49 & $7.48 \pm 1.24$ & $3.38 \pm 1.02^{\mathrm{a}, \mathrm{b}}$ & $1.89 \pm 1.12^{\mathrm{a}, \mathrm{b}}$ & $2.41 \pm 1.06^{\mathrm{a}, \mathrm{b}}$ & $2.57 \pm 1.16^{\mathrm{a}, \mathrm{b}}$ & $3.12 \pm 1.03^{\mathrm{a}, \mathrm{b}}$ \\
& $\mathrm{B}$ & 47 & $7.53 \pm 1.27$ & $5.11 \pm 1.13^{\mathrm{c}}$ & $5.17 \pm 1.07^{\mathrm{c}}$ & $5.13 \pm 1.12^{\mathrm{c}}$ & $6.12 \pm 1.03$ & $7.01 \pm 1.01$ \\
LKS & $\mathrm{A}$ & 49 & $42.4 \pm 19.4$ & $78.1 \pm 7.5^{\mathrm{a}, \mathrm{b}}$ & $74.7 \pm 5.2^{\mathrm{a}, \mathrm{b}}$ & $68.3 \pm 6.6^{\mathrm{a}, \mathrm{b}}$ & $83.1 \pm 4.6^{\mathrm{a}, \mathrm{b}}$ & $84.6 \pm 4.3^{\mathrm{a}, \mathrm{b}}$ \\
& $\mathrm{B}$ & 47 & $41.2 \pm 17.9$ & $61.1 \pm 5.3^{\mathrm{c}}$ & $62.2 \pm 4.7^{\mathrm{c}}$ & $54.1 \pm 6.2$ & $51.3 \pm 4.3$ & $43.2 \pm 6.1$ \\
\hline
\end{tabular}

Compared with group $\mathrm{B},{ }^{\mathrm{a}} \mathrm{P}<0.05$; after treatment compared with before treamtent of group $\mathrm{A},{ }^{\mathrm{b}} \mathrm{P}<0.05$; after treatment compared with before treatment of group $\mathrm{B},{ }^{\mathrm{c}} \mathrm{P}<0.05$.

Table III. Pain relief effective rates at each time points after treatment.

\begin{tabular}{|c|c|c|c|c|c|c|c|c|c|c|c|}
\hline \multirow[b]{2}{*}{ Groups } & \multirow[b]{2}{*}{ Cases } & \multicolumn{2}{|c|}{3 days } & \multicolumn{2}{|c|}{3 months } & \multicolumn{2}{|c|}{6 months } & \multicolumn{2}{|c|}{9 months } & \multicolumn{2}{|c|}{12 months } \\
\hline & & $\mathrm{n}$ & $\%$ & $\mathrm{n}$ & $\%$ & $\mathrm{n}$ & $\%$ & $\mathrm{n}$ & $\%$ & $\mathrm{n}$ & $\%$ \\
\hline A & 49 & 43 & $87.7^{\mathrm{a}}$ & 40 & $81.6^{\mathrm{a}}$ & 41 & $83.6^{\mathrm{a}}$ & 39 & $79.5^{\mathrm{a}}$ & 39 & $79.5^{\mathrm{a}}$ \\
\hline B & 47 & 30 & 63.8 & 25 & 51.3 & 22 & 46.8 & 18 & 38.2 & 18 & 38.2 \\
\hline
\end{tabular}

Compared with group $\mathrm{B},{ }^{\mathrm{a}} \mathrm{P}<0.05$; effective rates $=($ superior $+\operatorname{good}+$ effective cases $) /$ total cases.

The LKS was used to observe the improvement of daily life functions of patients including eight domains with a total score of 100: Limp (5-0), use of supports (5-0), locking (15-0), instability (25), pain (25-0), swelling (10-0), stair-climbing (10-0) and squatting (5-0 points) $(3,4)$.

Through follow-up by telephone or outpatient re-examination, scores were recorded for pain with VAS after patients did not take medicine for 3 days, and 3, 6, 9, and 12 months after treatment.

Criteria for therapeutic effect of pain relief: Excellent, VAS scores decreased by $>75 \%$; good, VAS scores decreased by $75-50 \%$; effective, VAS scores decreased by $50-25 \%$; ineffective, VAS scores decreased by $<25 \%$. Recording time, 1 and 3 months after treatment.

According to the following formula, the rate of effective pain relief $=[($ VAS scores before treatment - VAS scores after treatment)/VAS scores before treatment]x100\%.

Statistical analysis. Data were analyzed with SPSS 18.0 (SPSS, Inc., Chicago, IL, USA) software. A $\chi^{2}$ test was used for baseline analyses of parameters between the two groups; a paired t-test was used to compare intragroup differences before and after treatment; t-test was used to compare intergroup differences of measurement data; $\chi^{2}$ test was used to compare intergroup differences of enumeration data; and enumeration data are presented as rate or constituent ratio.

\section{Results}

Comparison of knee VAS scores. In group A, compared with before treatment, there were significant differences in knee
VAS at 3 days, and at 3, 6, 9, and 12 months after treatment $(\mathrm{P}<0.05)$. In group $\mathrm{B}$, there were significant differences in VAS at 3, 6, and 9 months $(\mathrm{P}<0.05)$ after treatment. Compared with group B, the improvements in pain relief in group A were significantly different at 3 days, and at 3, 6, 9, and 12 months $(\mathrm{P}<0.05)$ after treatment (Table II).

Comparison of knee LKS scores. Compared with before treatment, the LKS scores in group A were significantly different at 3 days and at 3, 6,9, and 12 months after treatment $(\mathrm{P}<0.05)$. In group $\mathrm{B}$, there were significant differences in LKS scores at 3 months after treatment $(\mathrm{P}<0.05)$. For improvement of pain relief, compared with group $\mathrm{B}$, the improvements of pain relief in group A were significantly different at 3 days, and at 3, 6, 9, and 12 months $(\mathrm{P}<0.05)$ after treatment (Table II).

In this study, it was observed that after post-treatment follow-up (telephone or home visit), in both A and group B, there were significant differences at 3 days, and at 3, 6, 9, and 12 months $(\mathrm{P}<0.05)$ (Table III).

\section{Discussion}

In addition to conservative treatment, there are currently many invasive treatments for knee OA (1), such as knee lavage, hyaluronic acid injection, glucocorticoid injection, arthroscopic synovial cleanup, and knee resurfacing, all of which have different indications and disadvantages (8). Knee lavage, glucocorticoid injection, and hyaluronic acid injection are applicable to mild and moderate OA, and can stabilize the intra-articular environment, increase articular lubrication, relieve synovitis, and enhance secreting hyaluronic acid $(9,10)$. 
Arthroscopic synovial cleanup and knee resurfacing are applicable to moderate and severe gonitis, and the latter is the final treatment for OA (11). However, because of the limited service life of prostheses and operative complications, the development of easier and more effective therapeutic methods is anticipated.

Nerve RF technology has been carried out locally for many years (12). By RF thermocoagulation denervation technology performed on the posterior rami of spinal nerves, most minor arthrogenous pain of the neck, shoulder and back can be effectively treated. Several clinical studies showed that RF thermocoagulation technology on pain points around the knee can be used to effectively alleviate pain around the knee (13-15). However, in the past, after applying similar technologies, we found that the range of $\mathrm{RF}$ was too wide, $\mathrm{RF}$ pain points were too many, the damage was greater, treatment duration was longer, patients experienced pain, and pain frequently recurred. Therefore, we assume that the pain caused by the knee can be controlled by breaking pathways of knee pain using nerve locator under accurate positioning to highly selective femoral ablation, lateral cutaneous nerve and knee saphenous nerve infrapatellar branch.

A nerve locator consists of casing and control circuit components (16). The casing is equipped with an ammeter plate and adjustment knob, while the control circuit contains the power supply, pulse signal generator and current regulating circuit. By applying DC pulse signals of certain frequencies on the nerve distribution area of the superficial fascia, the pulse signal generator can induce pain on corresponding positions. The resistance value of the potentiometer is gradually changed in the current regulating circuit and the intensity of the output current is reduced. When the minimum range that caused pain is found in the valley current, the accurate position of the cutaneous nerve is determined and the puncture point can be determined. By this nerve locator, accurate positioning for nerve stems is achieved using a simple circuit structure, avoiding pain caused by many punctures when searching for nerve stems. More objective indicators are obtained for patients with anatomic variations or those unable to accurately describe paresthesia, so the success rate of RF is greatly increased (14).

Most of the target nerves for highly selective peripheral nerve $\mathrm{RF}$ ablation of the knee are located in the superficial fascia layer, including the nervus cutaneus femoris lateralis, intermediate femoral cutaneous nerve, medial femoral cutaneous nerve and ramus infrapatellaris nervi sapheni (17). The nervus cutaneus femoris lateralis, as the terminal branch of L1 or L2 nerve roots, appear frequently and distribute to the upper and outer edges of the patella as well as the outer skin of the quadriceps tendon and superficial fascia, reaching the upper edges of the patella. Intermediate femoral cutaneous nerves stem from the femoral plexus and start from the femoral nerve groin, divide downward into two to three branches, and distribute to the skin of the quadriceps tendon, patella area, and superficial fascia. The lower medial femoral cutaneous nerves vary greatly, $38 \%$ of which are medial branches of femoral cutaneous nerves, while $60 \%$ are saphenous nerve branches (18), dominating the skin of the medial knee and superficial fascia. Saphenous nerves stem from posterior femoral branches, and separate into upper and lower branches at the lower ends of the fem interns, the lower branch of which is the ramus infrapatellaris nervi sapheni.

Nerves in circumpatellar soft tissue are located in the deep fascia layer (19), mainly including upper saphenous nerve branches, articular branches of patellar saphenous nerves, and articular branches of common peroneal nerves. Although articular cartilages may receive nutrition from these nerve branches, the metabolism of articular cartilage and surrounding tissues will not be affected as the RF thermocoagulation is only located in the superficial fascia layer.

The therapeutic effects for all patients in this study were as follows: Pain from knee OA was significantly relieved at the early period after treatment in groups A and B. However, half a year later, pain reoccurred and became severe in patients in group B, while in group A, the effect of treatment persisted, showing that highly selective nerve RF ablation is better for long-term analgesic effect. At 3 months after treatment, LKS scores in group B began to rebound, while in group A the scores remained lower, and the differences were statistically significant. This demonstrated that with the significant alleviation of pain, patients showed continuous improvements in knee motor functions and appeared to have no corresponding clinical manifestations of accelerated degeneration of articular cartilage.

In conclusion, with the use of highly selective peripheral nerve RF ablation, incoming pain signals were reduced around the knee, walking pain was effectively alleviated in the longterm, knee motor functions were improved, and quality of life was improved. This technology is easy to operate, very safe, and is worthy of being applied in hospitals of all levels. Additionally, the requirement for knee replacement may be reduced to some extent as the pain is greatly alleviated by this technology.

\section{Competing interests}

The authors declare that they have no competing interests.

\section{References}

1. Pendleton A, Arden N, Dougados M, Doherty M, Bannwarth B, Bijlsma JW, Cluzeau F, Cooper C, Dieppe PA, Gunther KP, et al: EULAR recommendations for the management of knee osteoarthritis: report of a task force of the Standing Committee for International Clinical Studies Including Therapeutic Trials (ESCISIT). Ann Rheum Dis 59: 936-944, 2000.

2. van Meurs JB: Osteoarthritis year in review 2016: Genetics, genomics and epigenetics. Osteoarthritis Cartilage 25: 181-189, 2017.

3. McCulloch K, Litherland GJ and Rai TS: Cellular senescence in osteoarthritis pathology. Aging Cell 16: 210-218, 2017.

4. Rathbun AM, Yau MS, Shardell M, Stuart EA and Hochberg MC: Depressive symptoms and structural disease progression in knee osteoarthritis: Data from the osteoarthritis initiative. Clin Rheumatol 36: 155-163, 2017.

5. Dimitroulas T, Lambe T, Klocke R, Kitas GD, Duarte RV: Biologic drugs as analgesics for the management of osteoarthritis. Semin Arthritis Rheum 46: 687-691, 2017

6. Herrero-Beaumont G, Roman-Blas JA, Bruyère $O$, Cooper C, Kanis J, Maggi S, Rizzoli R and Reginster JY: Clinical settings in knee osteoarthritis: Pathophysiology guides treatment. Maturitas 96: 54-57, 2017.

7. Papalia R, Del Buono A, Osti L, Denaro V and Maffulli N: Meniscectomy as a risk factor for knee osteoarthritis: a systematic review. Br Med Bull 99: 89-106, 2011. 
8. White DK and Master H: Patient-reported measures of physical function in knee osteoarthritis. Rheum Dis Clin North Am 42 : 239-252, 2016

9. Fatimah N, Salim B, Raja EU and Nasim A: Predictors of response to intra-articular steroid injections in patients with osteoarthritis of the knee joint. Clin Rheumatol 35: 2541-2547, 2016.

10. Lapane KL, Liu SH, Dubé CE, Driban JB, McAlindon TE and Eaton CB: Factors associated with the use of hyaluronic acid and corticosteroid injections among patients with radiographically confirmed knee osteoarthritis: A retrospective data analysis. Clin Ther 39: 347-358, 2017.

11. Ali A, Lindstrand A, Nilsdotter A and Sundberg M: Similar patient-reported outcomes and performance after total knee arthroplasty with or without patellar resurfacing. Acta Orthop 87: 274-279, 2016

12. Vas L, Pai R, Khandagale N and Pattnaik M: Pulsed radiofrequency of the composite nerve supply to the knee joint as a new technique for relieving osteoarthritic pain: A preliminary report. Pain Physician 17: 493-506, 2014.

13. Udupi BP, Chouhan RS, Dash HH, Bithal PK and Prabhakar H: Comparative evaluation of percutaneous retrogasserian glycerol rhizolysis and radiofrequency thermocoagulation techniques in the management of trigeminal neuralgia. Neurosurgery 70 : 407-412; discussion 412-403, 2012.

14. Bhatia A, Peng P and Cohen SP: Radiofrequency procedures to relieve chronic knee pain: An evidence-based narrative review. Reg Anesth Pain Med 41: 501-510, 2016.
15. Kesikburun S, Yaşar E, Uran A, Adigüzel E and Yilmaz B: Ultrasound-guided genicular nerve pulsed radiofrequency treatment for painful knee osteoarthritis: A preliminary report. Pain Physician 19: 751-759, 2016.

16. Wellehan JF, Gunkel CI, Kledzik D, Robertson SA and Heard DJ: Use of a nerve locator to facilitate administration of mandibular nerve blocks in crocodilians. J Zoo Wildl Med 37: 405-408, 2006.

17. Barton RS, Ostrowski ML, Anderson TD, Ilahi OA and Heggeness MH: Intraosseous innervation of the human patella: A histologic study. Am J Sports Med 35: 307-311, 2007.

18. Bjurlin MA, Davis KE, Allin EF and Ibrahim DT: Anatomic variations in the lateral femoral cutaneous nerve with respect to pediatric hip surgery. Am J Orthop (Belle Mead NJ) 36: 143-146, 2007.

19. Hirasawa Y, Okajima S, Ohta M and Tokioka T: Nerve distribution to the human knee joint: Anatomical and immunohistochemical study. Int Orthop 24: 1-4, 2000. 